\title{
Equilibrium polymerization of cyclic carbonate oligomers. II. Role of multiple active sites
}

\author{
P. Ballone \\ Institut für Festkörperforschung, Forschungszentrum Jülich, D-52425 Jülich, Germany \\ and Università degli Studi di Messina, Dipartimento di Fisica, Contrada Papardo, I-98166 Messina, Italy \\ R. O. Jones ${ }^{a)}$ \\ Institut für Festkörperforschung, Forschungszentrum Jülich, D-52425 Jülich, Germany
}

(Received 30 November 2001; accepted 8 February 2002)

\begin{abstract}
Ring opening polymerization of bisphenol A polycarbonate is studied by Monte Carlo simulations of a model comprising a fixed number of Lennard-Jones particles and harmonic bonds [J. Chem. Phys. 115, 3895 (2001)]. Bond interchanges produced by a low concentration $\left(0.10 \% \leqslant c_{a}\right.$ $\leqslant 0.36 \%$ ) of chemically active particles lead to equilibrium polymerization. There is a continuous transition in both 2D and 3D from unpolymerized cyclic oligomers at low density to a system of linear chains at high density, and the polymeric phase is much more stable in three dimensions than in two. The steepness of the polymerization transition increases rapidly as $c_{a}$ decreases, suggesting that it is discontinuous in the limit $c_{a} \rightarrow 0$. The transition is entropy driven, since the average potential energy increases systematically upon polymerization, and there is a steady decline in the degree of polymerization as the temperature is lowered. The mass distribution functions for open chains and for rings are unimodal, with exponentially decaying tails that can be fitted by ZimmSchulz functions and simpler exponential forms. (C) 2002 American Institute of Physics.
\end{abstract}

[DOI: $10.1063 / 1.1466834]$

\section{INTRODUCTION}

The bonds defining the structure of most polymers are formed during a short polymerization stage and remain unchanged because of kinetic or chemical constraints. Some polymers, however, retain their ability to form and break bonds. Their structures depend on the competition between polymerization and depolymerization reactions, and they can change if an external perturbation is applied. Equilibrium or "living" polymers ${ }^{1}$ include inorganic and organic systems (such as sulfur and $\alpha$-methylstyrene, respectively) and are particularly important in biological processes, where complex structures can be assembled or disassembled in response to very small changes in the environment. They also play a crucial role in understanding polymerization in general, because the presence of equilibrium allows the application of standard methods of statistical mechanics.

The current interest in equilibrium polymers is reflected in the increasing number of experimental and theoretical publications. ${ }^{2}$ Early theoretical analyses were based on approximate analytic methods, mainly of the mean-field and kinetic rate equations types, ${ }^{3,4}$ and computer simulations of idealized models are now providing a wealth of information on scaling relations and other universal properties. Several studies $^{5}$ have been based on the bond-fluctuation model, ${ }^{6}$ in which particles on a lattice can form up to a given number of bonds within a fixed distance. Polymerization proceeds via the condensation and evaporation of monomers at the poly-

\footnotetext{
a) Author to whom correspondence should be addressed. Electronic mail: r.jones@fz-juelich.de
}

mer boundary, defined by the monomers whose bonds are not saturated. More recently, continuum models using simple potential energy functions for the intra- and interchain interactions have been introduced. ${ }^{7}$ Such calculations are usually restricted to flexible polymers that do not form closed structures (rings in the case of linear polymers), ${ }^{8}$ and simulation results agree well with the predictions of mean field and renormalization group theories. ${ }^{9}$

Recently, we performed Monte Carlo (MC) simulations for a model that differs from previous ones in both the interparticle Hamiltonian and the polymerization/ depolymerization mechanism. The calculations were motivated by the ring opening polymerization of bisphenol A polycarbonate (BPA-PC), ${ }^{10}$ where the reactant (a condensed phase of cyclic oligomers) gives rise to high molecular weight chains in the presence of a suitable nucleophilic catalyst. The first step of the reaction is illustrated in Fig. 1 for an alkali metal phenoxide molecule $(\mathrm{MOPh}$, where $\mathrm{M}$ is $\mathrm{Li}$ or $\mathrm{Na}$ ) reacting with a $\mathrm{PC}$-tetramer. This process changes neither the number nor nature of the covalent bonds, and the results of density functional (DF) calculations ${ }^{11}$ agree with the experimental finding that reactants and products have virtually the same energy. ${ }^{10}$ The reaction barrier can be reduced below a few $\mathrm{kcal} / \mathrm{mol}$ by a suitable choice of $\mathrm{M}$, and the product (Fig. 1) has the same active head as in MOPh. A steady supply of oligomers would allow this reaction to be repeated indefinitely to yield a long polymer chain, but the growth is limited in practice by several processes: (a) after the first step the active head may react with its own tail, shedding rings of differing sizes, (b) competition between 


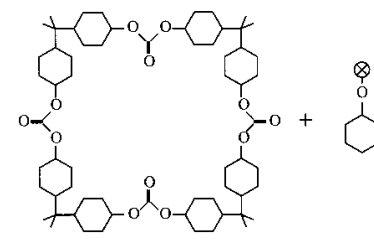

(b)

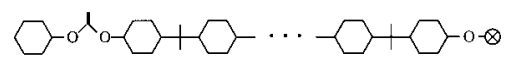

FIG. 1. Schematic view of the first step in the ring opening polymerization of BPA-PC. The active site is an electropositive metal atom, identified by a cross.

active chains will limit the supply of oligomers, (c) the intersection of two active chains may result in the interchange of their tails (see Fig. 2), eventually leading to chains that have the same average properties.

Our model ${ }^{12}$ reproduces all these features and allowed us to perform an extensive investigation of polymerization as a function of dimensionality (2D or 3D), density, and temperature $T$. We focused on the limit of vanishing catalyst concentration, with a single active particle in a system of 2500 tetramers. The formation of long chains in most of the simulations was driven by the entropy associated with the distribution of covalent bonds. Polymerization occurs in 2D via a weakly first order transition, whereas other models predict a continuous transition.

Here we extend our earlier study to concentrations of active particles $(0.1 \%-0.36 \%)$ corresponding to those of catalysts in real systems. The presence of multiple active heads in samples of the same size improves the statistics, reduces finite size effects, and allows us to study in detail the size distribution of the polymers as a function of the concentration of active heads, density, and temperature. The polymerization transition as a function of density is continuous, becoming discontinuous as the catalyst concentration vanishes. We confirm the existence of the polymerization transition in three dimensions.

The strict conservation of the number of bonds makes our model particularly suitable to describe equilibrium polymers with strong intrachain bonds that neither form nor break at moderate temperatures, but which can be interchanged in pairs under the action of a catalyst. Most previous studies of equilibrium polymerization have relied on models with a fluctuating population of unsaturated particles, whose average number is determined by thermal equilibrium. These models are then more appropriate for systems with fairly low intrachain bond energies.

\section{THE MODEL AND THE COMPUTATIONAL METHOD}

BPA-PC structural units are represented in the model of Ref. 12 by Lennard-Jones (LJ) particles, ${ }^{13}$ and the covalent bonds in the polymer backbone are described by harmonic springs. Each particle forms one or two harmonic bonds, so that the system comprises open chains or rings ${ }^{14}$ without branching. The potential energy of a collection of (N/4) cyclic tetramers, for example, is (a)

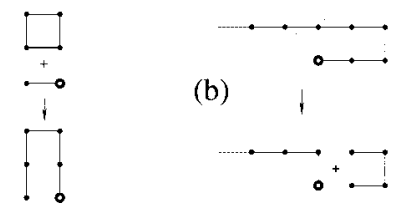

(c)
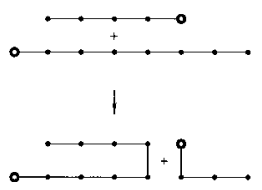

FIG. 2. Bond-interchange processes leading to equilibrium polymerization. Active heads are denoted by large empty circles.

$$
\begin{aligned}
V\left(\mathbf{r}_{1}, \ldots, \mathbf{r}_{\mathbf{N}}\right) \\
=\epsilon \sum_{i>j}^{N^{\prime}}\left\{\left(\frac{\sigma}{\left|\mathbf{r}_{i}-\mathbf{r}_{j}\right|}\right)^{12}-\left(\frac{\sigma}{\left|\mathbf{r}_{i}-\mathbf{r}_{j}\right|}\right)^{6}\right\} \\
\quad+\frac{1}{2} k \sum_{\alpha=1}^{(N / 4)}\left\{\left(\mathbf{r}_{4 \alpha-3}-\mathbf{r}_{4 \alpha}\right)^{2}+\left(\mathbf{r}_{4 \alpha-3}-\mathbf{r}_{4 \alpha-2}\right)^{2}\right. \\
\left.+\left(\mathbf{r}_{4 \alpha-2}-\mathbf{r}_{4 \alpha-1}\right)^{2}+\left(\mathbf{r}_{4 \alpha-1}-\mathbf{r}_{4 \alpha}\right)^{2}\right\},
\end{aligned}
$$

where the prime on the first sum indicates that the LJ interaction is absent when $i$ and $j$ are connected by the harmonic potential.

The units of length and energy are $\sigma$ and $\epsilon$, respectively, and the density is described by the packing fraction $\eta(\eta$ $=\pi \rho \sigma^{2} / 4$ in $2 \mathrm{D}, \eta=\pi \rho \sigma^{3} / 6$ in $\left.3 \mathrm{D}\right)$. The choice of $k(k$ $=3 \epsilon$ ) leads to very floppy molecules and allows us to sample efficiently both the intra- and intermolecular degrees of freedom. Angle bending and torsion contributions are neglected, so that we simulate fully flexible polymers.

The reaction is initiated by introducing $N_{a}\left(N_{a}=10,16\right.$, $25,36)$ active particles. These interact with the rest of the system via the same potentials as the other particles, but they mimic the behavior of the catalytic head in MOPh because (a) they form one and only one bond, and (b) bond exchanges occur only when at least one of the bonds involves an active particle. The different bond configurations are sampled by identifying at regular intervals the nearest neighbor of an active particle (excluding the particle connected to it by an intramolecular bond) and linking this pair and two of their covalently bonded neighbors as shown in Fig. 2. The interchange conserves the total number of bonds and is accepted or rejected on the basis of the changes in potential energy and $T$, using the Metropolis algorithm to approach the Boltzmann distribution for the potential energy.

The potential energy of the system is sampled by a (constant volume) MC method, in which we attempt to translate either single particles or whole molecules with relative probability and steps chosen to achieve an acceptance ratio of $\sim 0.5$ for both types of move. The interchange of intermolecular bonds is attempted every $N$ single particle steps, with $N$ of the order of the number of particles in the system. 
All simulations were started from well equilibrated samples of 2500 cyclic tetramers plus $N_{a}$ dimers $\left(N_{a}=10\right.$, $16,25,36)$ with one active particle each. The bond exchange rules do not allow a particle to change the number of its covalent bonds. The number of singly bonded particles $\left(2 N_{a}\right.$, half active and half regular) and the number of open chains $\left(N_{a}\right)$ then remain constant as the system evolves, while the number of rings fluctuates. In other words, we simulate collections of $10000+2 N_{a}$ particles and 10000 $+N_{a}$ bonds in thermal equilibrium, under the restrictions of fixed numbers of chain terminations (i.e., singly bonded particles) and open chains. The separation into "active" and "regular" chain terminations reflects kinetic properties alone: Bond exchanges can occur when one active (catalyst) particle is involved, and are kinetically frozen otherwise. This distinction cannot affect the equilibrium properties, which depend only on the relative concentration of singlyand doubly-bonded particles, but it describes the ringopening polymerization of BPA-PC and corresponds to a specific choice of the attempt rate for the bond interchange MC moves.

The constant number of bonds and the corresponding conservation of the number of chain terminations (singly bonded particles) are the major differences between our model and those used in previous simulation studies. In the continuum model of Ref. 7, for instance, the average number of unsaturated particles depends on temperature via a parameter $J$ that measures the energy required to break a bond. Comparison of our results with those of Ref. 7 will then allow purely statistical effects (related to the multiplicity of chains and rings of different length) to be separated from potential energy effects arising from changes in the number of bonds.

Open chains may have zero, one or two active terminations during the simulation, with probabilities $1 / 4,1 / 2$, and $1 / 4$, respectively. Monitoring the number of active terminations or the number of tetramers provides useful checks of equilibration. The initial excess of tetramers is reduced progressively, and their number fluctuates without drift at equilibrium with a concentration between those of trimers and pentamers. Equilibrium with respect to translational degrees of freedom was monitored by computing the average energy and structural properties such as the pair distribution function. For all systems, equilibration lasted more than $2 \times 10^{5}$ MC steps/particle, with an equal number $\left(>2 \times 10^{5}\right)$ of bond interchange attempts. Statistics have been accumulated on additional runs of equal length.

The diffusion and viscosity coefficients were computed by microcanonical molecular dynamics (MD) runs for systems of fixed bonding configuration, ${ }^{15}$ starting from atomic positions equilibrated by $\mathrm{MC}$ and random velocities selected from a Gaussian distribution. Together with $\sigma$ and $\epsilon$, the unit mass (the mass of the monomer $m$ ) determines the unit of time $\tau=\sigma(m / \epsilon)^{1 / 2}$. The equations of motion have been integrated using the velocity Verlet algorithm with a time step of $0.5 \times 10^{-2} \tau . M$ is the mass of the polymer chain and numerically equal to the number of monomers.

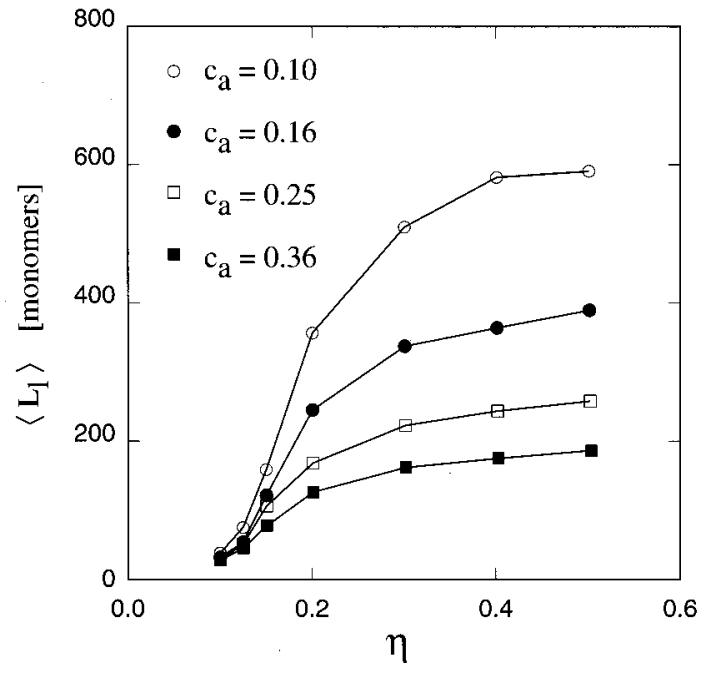

FIG. 3. Average length of open chains as a function of packing fraction $\eta$ for different concentrations (in \%) of active particles. Simulations in 2D.

\section{RESULTS}

Simulations have been performed for a wide range of densities $(0.1<\eta<0.5$ in $2 \mathrm{D}, 0.0125<\eta<0.5$ in $3 \mathrm{D}$, with some computations up to $\eta=1$ for $2 \mathrm{D}$ and $3 \mathrm{D}$ ), temperature $(0.2 \leqslant T \leqslant 3)$, and number of active particles $\left(10 \leqslant N_{a} \leqslant 36\right.$, corresponding to concentrations $0.1 \%<c_{a}<0.36 \%$ ) in two and three dimensions. The results for $2 \mathrm{D}$ are reported first, and then we describe similarities and differences observed in 3D. The results do not depend strongly on $T$, and we focus on high- $T$ data $(T=3 \epsilon)$, for which the phase space is sampled very efficiently and the statistics are better than at lower $T$. Open chains and rings have dramatically different properties in polymerized samples and are discussed separately. Polymerization is always due to the massive growth of the open chains, while the average size of rings increases only slightly, despite the appearance of a few large rings. The fraction of total mass represented by open chains is used to monitor the degree of polymerization in the system.

\section{A. The polymerization transition in $2 \mathrm{D}$}

Addition of dimers with active heads gives rise to continuing bond interchanges in all 2D samples, forming long open chains at high densities. These chains grow slowly at low density $(\eta<0.15)$, evolving almost independently and with an average length $\left\langle L_{l}\right\rangle$ that depends very weakly on $c_{a}$. Open chains represent a large fraction of the total mass at high density, and the competition between them to incorporate the residual rings limits their average length as $N_{a}$ increases. In this regime, the fraction of the total mass in the open chains (typically 50\%-65\%) depends only weakly on $c_{a}$, and the average length $\left\langle L_{l}\right\rangle$ scales as $1 / c_{a}$. These features are apparent in Fig. 3, which shows the average length $\left\langle L_{l}\right\rangle$ of open chains as a function of $\eta$ for different concentrations of active particles. Figure 4 shows the corresponding fraction of the total mass in open chains.

In all cases, but especially in the low $c_{a}$ limit, the $\left\langle L_{l}\right\rangle(\eta)$ curves show a steep rise at $\eta \sim 0.15$, which can be viewed as a rounded transition to the polymeric state. To 


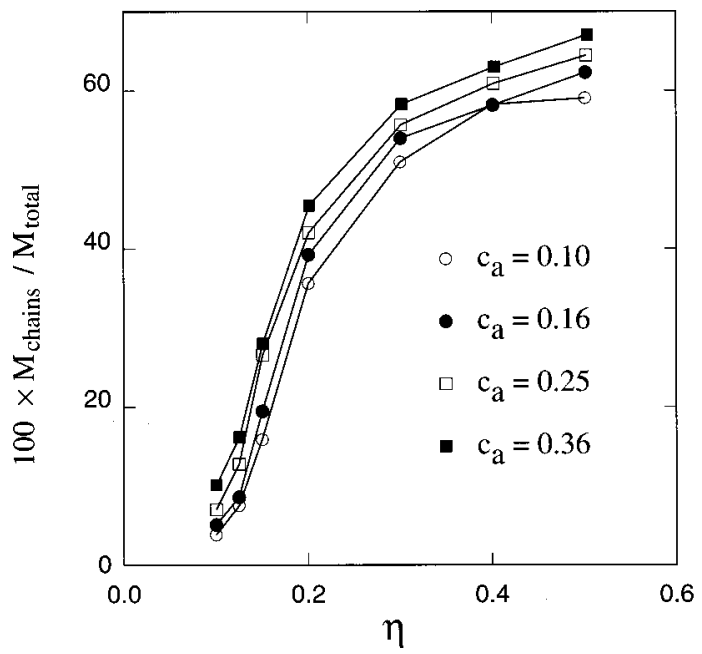

FIG. 4. Percentage of the total mass in open chains as a function of packing fraction $\eta$. Simulations in 2D with different concentrations $c_{a}$ (in $\%$ ) of active particles.

quantify this observation, we fit each S-shaped curve of Fig. 3 with a Padé ratio, ${ }^{16}$

$$
\begin{aligned}
\left\langle L_{l}\right\rangle(\eta) & \sim\left(\frac{N_{\mathrm{tot}}}{N_{a}}\right) \frac{a \eta+b \eta^{2}+c \eta^{3}}{1+d \eta+e \eta^{2}+c \eta^{3}} \\
& =\left(\frac{1}{c_{a}}\right) \frac{a \eta+b \eta^{2}+c \eta^{3}}{1+d \eta+e \eta^{2}+c \eta^{3}} .
\end{aligned}
$$

The value $\bar{\eta}$ at which the curvature of the interpolated function changes sign is our estimate of the transition point, while the slope of $\left\langle L_{l}\right\rangle$ at $\bar{\eta}$ is a measure of its steepness. The steepness increases rapidly and without apparent upper bound as $c_{a}$ decreases (Fig. 5), suggesting that the transition becomes discontinuous in the limit that $c_{a}$ vanishes. On the other hand, an increasing number of medium sized chains ( $\sim 50-100$ monomers each) is present in the low density phase as $N_{a}$ increases. They cooperate to trigger the polymerization transition, as is apparent from the systematic decrease of $\bar{\eta}$ with increasing $N_{a}$. Although effected by larger

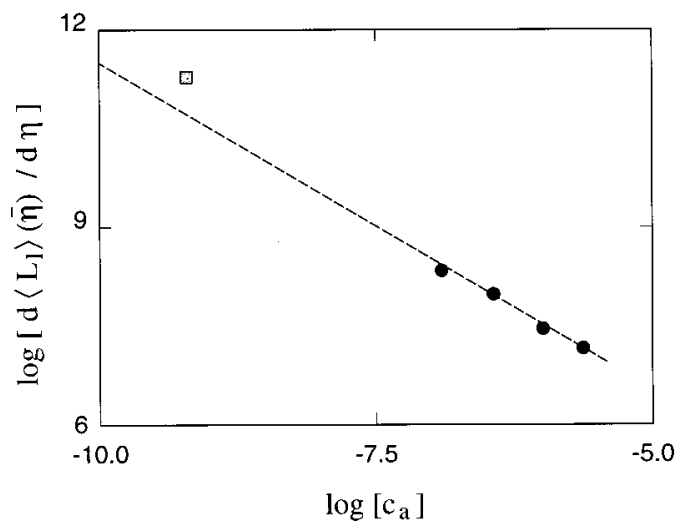

FIG. 5. Logarithmic plot of $d / d \eta\left\langle L_{l}\right\rangle(\bar{\eta})$ computed at the inflection point $\bar{\eta}$ of $\left\langle L_{l}\right\rangle(\eta)$ as a function of the concentration $c_{a}$ of active particles. The empty square shows the result of Ref. $12\left(c_{a}=0.01 \%\right)$.

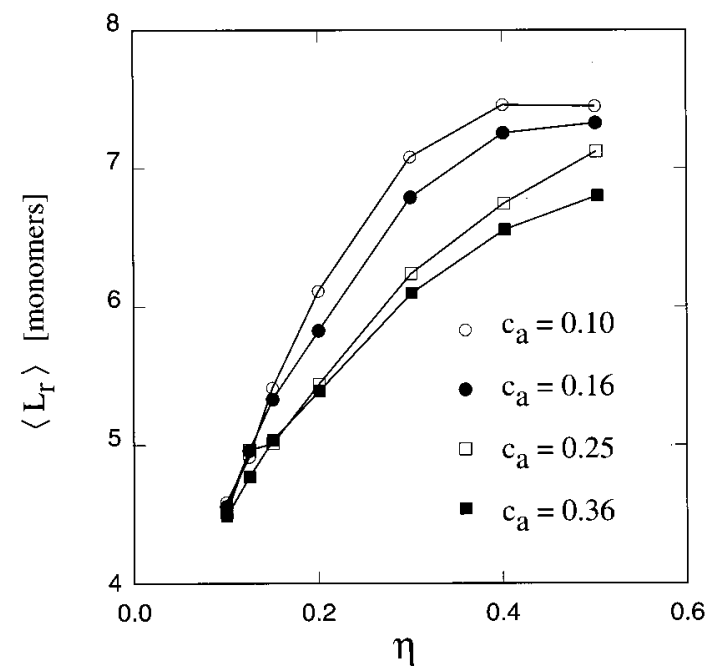

FIG. 6. Average length of rings in 2D systems with different concentrations of active particles.

statistical and finite size errors, the data for $c_{a}=0.01$ (Ref. 12) are consistent with those computed here for higher concentrations.

The average length $\left\langle L_{r}\right\rangle$ of rings as a function of $\eta$ is shown in Fig. 6 for different $c_{a}$ values. The shape of these curves is reminiscent of that for $\left\langle L_{l}\right\rangle(\eta)$, but the scale of $\left\langle L_{r}\right\rangle$ and its variations with $\eta$ and $c_{a}$ are much smaller. As discussed below, the increase of $\left\langle L_{l}\right\rangle(\eta)$ is induced by the growth of long open chains as the density increases, giving rise occasionally to rings of nearly the same length.

The equilibrium size distributions of rings and chains are characterized by the mass-fraction distributions $P_{r}(L)$ and $P_{l}(L)$, defined as the percentage of monomers belonging to a ring or a chain, respectively, of length $L$. These quantities are preferable here to the size distributions $C_{l}(L)$ and $C_{r}(L)$, which emphasize the role of the (relatively few) $N_{a}$ long chains that represent a sizable fraction of the total mass. These distributions are simply related by $P_{l}(L)=L C_{l}(L)$ and $P_{r}(L)=L C_{r}(L)$. The distribution functions discussed below are normalized so that

$$
\sum_{L} P_{l}(L)=\sum_{L} P_{r}(L)=100 .
$$

The dependencies of $P_{l}(L)$ and $P_{r}(L)$ on $\eta$ and $N_{a}$ are shown in Fig. 7 and Fig. 8, respectively. The mass fraction distribution for chains is always relatively broad, and the Zimm-Schulz distribution,

$$
P_{l}(L) \propto L^{\gamma} \exp \left[-\gamma L /\left\langle L_{l}\right\rangle\right]
$$

provides a fair fit to all curves, with an exponent $(1.1<\gamma$ $<1.4)$ close to the value predicted by analytic theories $\gamma$ $=43 / 32 .{ }^{4}$ The Zimm-Schulz fit may reflect little more than the existence of a $P_{l}(L)$ that is unimodal, broad and relatively featureless, decays exponentially for high $L$, and scales simply with $\eta$ and $c_{a} \cdot{ }^{17}$ In fact, a similar fit is provided by other expressions, including $P_{l}(L) \propto L$ $\times \exp \left[-\gamma L /\left\langle L_{l}\right\rangle\right]$ and $P_{l}(L) \propto L^{\gamma} \exp \left[-L /\left\langle L_{l}\right\rangle\right]$. The wide distribution of sizes is reflected in the standard deviation for $L_{l}$, which is $80 \%$ of $\left\langle L_{l}\right\rangle$, almost independent of $\eta$ and $c_{a}$. 


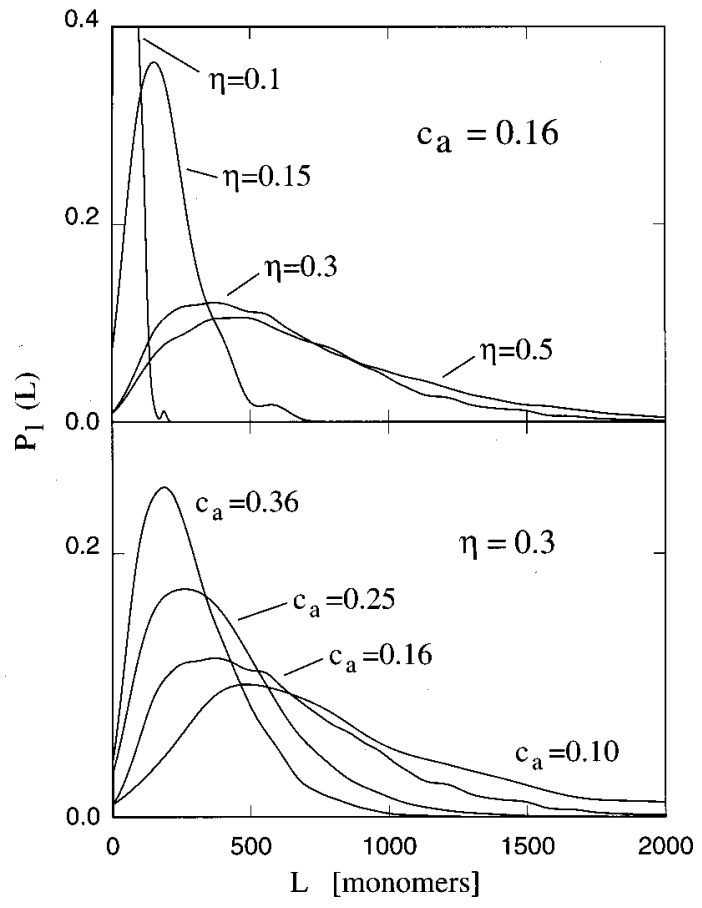

FIG. 7. Mass-fraction distribution functions for open chains in 2D systems. (Upper panel) results for different densities at fixed concentration of active particles $\left(c_{a}=0.16 \%\right)$. (Lower panel) results for different values of $c_{a}$ at fixed packing fraction $(\eta=0.3)$.

A more quantitative analysis shows that with decreasing $c_{a}$ the quality of the fit becomes progressively worse, partly because of increased statistical noise at low concentrations. Part of the discrepancy seems to reflect a real and progressive deviation of $P_{l}(L)$ from the simple forms used in the fit, particularly in the high $L$ tail. This might be due to finite size effects, which reduce the probability for chains whose length exceeds $N / N_{a}$, but a definite conclusion would require a sequence of simulations for different system sizes.

The distribution $P_{r}(L)$ for rings changes very little over the range of $\eta$ and $c_{a}$ considered (see Fig. 8). The increase of $\left\langle L_{r}\right\rangle$ with increasing $\eta$ apparent in Fig. 6 arises from a broad background that is difficult to identify on a linear scale, but which extends over the entire range of the much wider distribution $P_{l}(L)$. The clearest signature of the background is the progressive decrease in the height of the peak of $P_{r}(L)$ with increasing $c_{a}$, while the peak position $\bar{L}=4-5$ does not change [see Fig. 8(a)]. The enhancement of this background as the density increases is also reflected in a sharp increase in the standard deviation of $\left\langle L_{r}\right\rangle$ from $\sim 66 \%$ of $\left\langle L_{r}\right\rangle$ for $\eta \sim 0.1$ to more than $200 \%$ for $\eta \sim 0.4$. The tail in $P_{r}(L)$ for large $L$ reflects the fact that long chains have a small but non-vanishing probability of producing large rings when the active head reacts with its own tail close to the opposite termination [see Fig. 2(b)]. The peak of $P_{r}(L)(L$ $\leqslant 50)$ is well approximated by $P_{r}(L) \propto L \exp \left[-L /\left\langle L_{r}\right\rangle\right]$. This result is qualitatively different from mean field predictions ${ }^{18}$ and simulation data ${ }^{7}$ for models in which the number of bonds is not constrained, but fluctuates in thermal equilibrium, giving rise to a singular distribution $P_{r}(L)$ $\propto \exp \left[-L /\left\langle L_{r}\right\rangle\right] / L$ for small sizes.

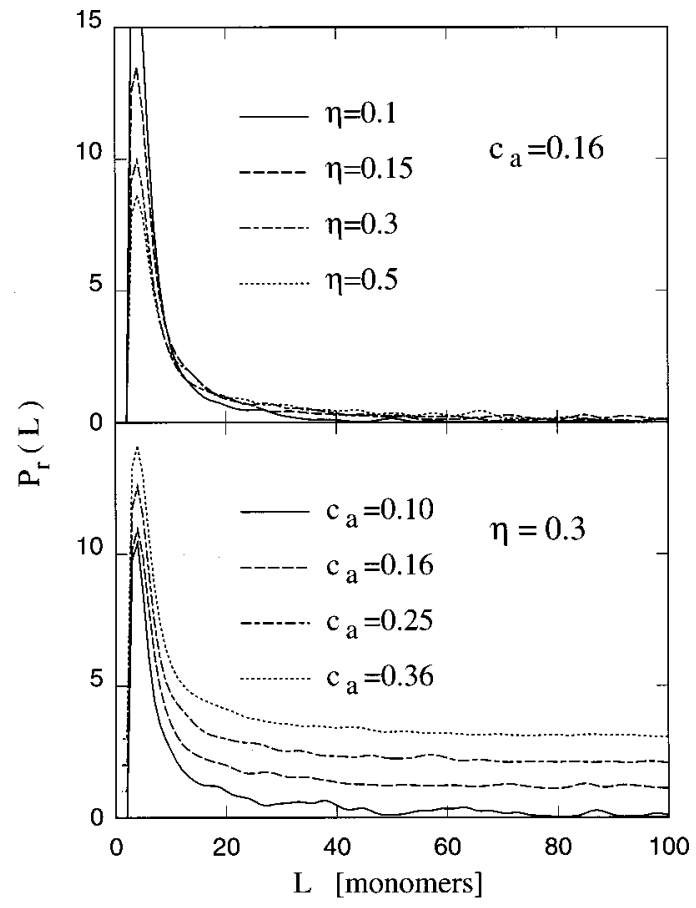

FIG. 8. Mass-fraction distribution functions for rings in 2D systems. (Upper panel) results for different densities at fixed concentration of active particles $\left(c_{a}=0.16 \%\right)$. (Lower panel) results for different values of $c_{a}$ at fixed packing fraction $(\eta=0.3)$. These curves are so similar that their ordinates have been shifted successively by one unit to improve clarity.

\section{B. Temperature dependence and thermodynamic properties}

For models in which the number of unsaturated particles is determined by thermal equilibrium, mean field theory predicts that the $T$-dependence of the average polymer length is $\langle L\rangle \sim \exp (J / 2 T)$, where $J$ is the energy required to break a chemical bond. ${ }^{19}$ For stable molecules $(J>0)$, the degree of polymerization decreases with increasing $T$. Potential energy cannot be the driving force for polymerization in our model, as can be seen in the $T$-dependence of the degree of polymerization for a system with $N_{a}=16, \eta=0.3$ and $0.3 \leqslant T$ $\leqslant 3$. The system remains homogeneous as $T$ is lowered to $T \sim 0.5$, while for $T \leqslant 0.4$ a high density phase comprising mainly long rings coexists with a dilute phase with many rings of low molecular (Fig. 9).

Although all simulations resulted in systems on the polymer side of the polymerization line for all $T$, temperature has a significant effect on the degree of polymerization. This is shown in Fig. 10, where we plot the fraction of the mass contributed by open chains as a function of $T$. For $T \leqslant 0.4$ the lower tendency to polymerization is partly compensated by condensation that leads to islands of higher density and enhanced polymerization. The average length of rings is almost constant, and the progressive increase at low $T$ in the relative fraction of the total mass in rings is due to a significant increase in their average number.

This trend can be related to the temperature dependence of the extension of the chains, which can be monitored by computing the radius of gyration, 


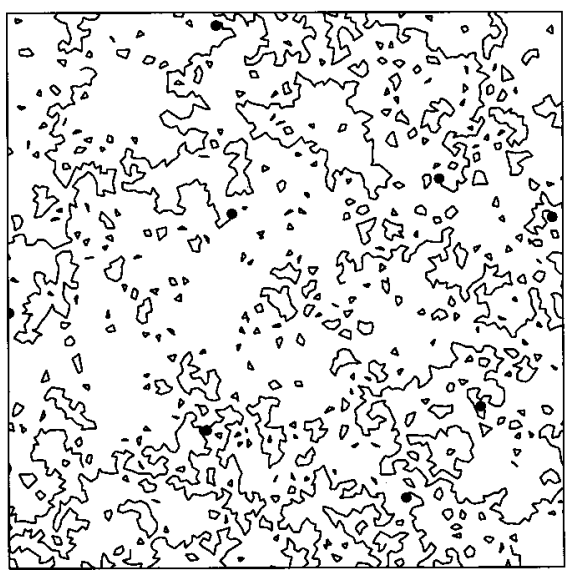

FIG. 9. Snapshot of a small portion of a 2D system in the two-phase coexistence region $\left(T=0.4, \eta=0.3\right.$, and $\left.c_{a}=0.16 \%\right)$ with condensed islands comprising polymerized chains and a low density fluid phase enriched in short rings.

$$
R_{g}=\sqrt{\frac{1}{2 N^{2}} \sum_{i \neq j}^{N}\left\langle\left(\mathbf{x}_{\mathbf{i}}-\mathbf{x}_{\mathbf{j}}\right)^{2}\right\rangle} .
$$

The chains are coiled (i.e., $R_{g} \sim N$ ) for all $T$ considered in our simulations, but $R_{g}$ decreases with decreasing $T$, making more effective the only process that gives rise to rings [Fig. 2(b)]. The origin of both effects (the reduction of $R_{g}$ and the enhancement of the ring population) must arise from the temperature dependence of the balance between energy and entropy underlying the polymerization transition.

Temperature-induced changes in the mass distribution probability follow the Zimm-Schulz form observed at $T$ $=3$, with an exponent $\gamma$ that is almost constant. As a result, all probability distributions can be folded into a single curve by rescaling with the average chain or ring lengths.

Additional computations at $T=1$ with different densities and $c_{a}$ values show that there is little change in the density $\bar{\eta}$ at which polymerization takes place, in spite of the reduction in the degree of polymerization as $T$ is lowered. The qualitative features of the transition also change little; the curve $\left\langle L_{l}(\eta)\right\rangle$ is continuous for every choice of $N_{a}$, but its steepness at $\bar{\eta}$ grows with decreasing $N_{a}$, indicating a first order transition in the limit of a vanishing concentration of active particles.

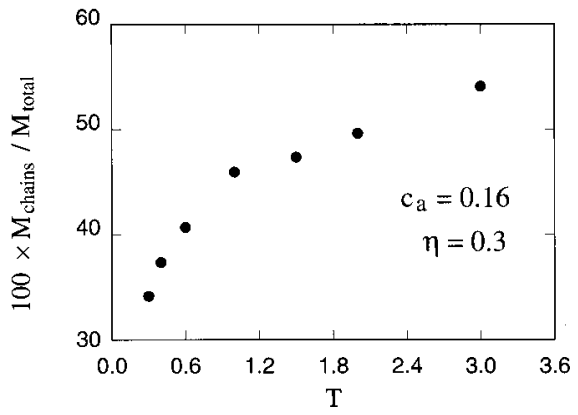

FIG. 10. Fraction of the total system mass in open chains as a function of temperature in $2 \mathrm{D}$ systems.

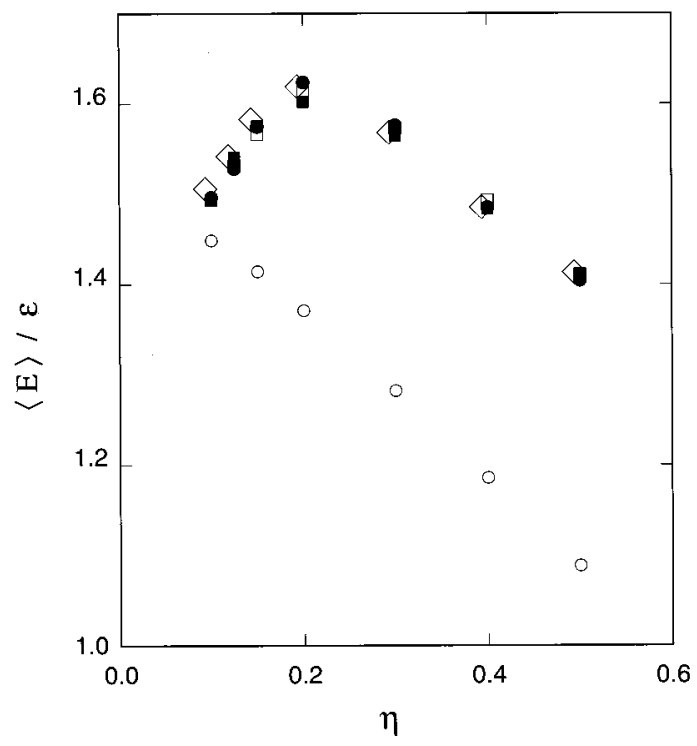

FIG. 11. Average potential energy per particles in 2D systems as a function of packing fraction $\eta$ at $T=3$. Solid dots, $c_{a}=0.10 \%$; filled squares, $c_{a}$ $=0.16 \%$; empty squares, $c_{a}=0.25 \%$; diamonds, $c_{a}=0.36 \%$; empty circles, systems at fixed bonding $\left(c_{a}=0\right)$ made by cyclic tetramers.

As in the case $N_{a}=1,{ }^{12}$ the presence of several active particles during polymerization leads to an increase in the average potential energy, which is systematically higher at equilibrium than in the unpolymerized state. This is shown in Fig. 11, where the equilibrium value of the potential energy is plotted as a function of $\eta$ (at $T=3$ ) for the unreacted samples $\left(c_{a}=0\right)$, and for samples containing active particles. Similar results are obtained at lower $T$, and the difference between unpolymerized and polymerized samples depends only slightly on $c_{a}$ at all $T$. These results confirm that polymerization in these systems must be entropy-driven.

Dynamical coefficients (diffusion coefficient $D$ and viscosity coefficient $s$ ) have been computed by MD at fixed bonding configuration. They depend only weakly on $c_{a}$ and are very similar to those of Ref. 12 for $c_{a}=0.01 \%$. Both quantities depend little on the average length of the open chains (which depends on $c_{a}$ ) in the polymeric phase. Diffusion is due mainly to the residual population of oligomers (mainly rings), which decreases only slowly with increasing $c_{a}$, and the viscosity is affected little by the presence of a few chain terminations, once the polymerized chains are close to each other and cover most of the sample.

We have also analyzed the mobility of the particles found during the $\mathrm{MC}$ simulation with the bond interchange sampling. In analogy with the real time analysis, we compute the average distance between two configurations in the Markov chain separated by $\tau$ MC steps,

$$
\Delta^{2}(\tau)=\sum_{i=1}^{N_{\text {tot }}}\left\langle\left|\mathbf{r}_{\mathbf{i}}(\tau)-\mathbf{r}_{\mathbf{i}}(0)\right|^{2}\right\rangle
$$

where the average is taken over different origins $(\tau=0)$ of the MC sequence. As in its MD counterpart, $\Delta^{2}(\tau)$ show a linear behavior at large $\tau$, with a linear coefficient that can be considered the MC analogy of the diffusion coefficient. This quantity cannot be converted into a real time diffusion coef- 


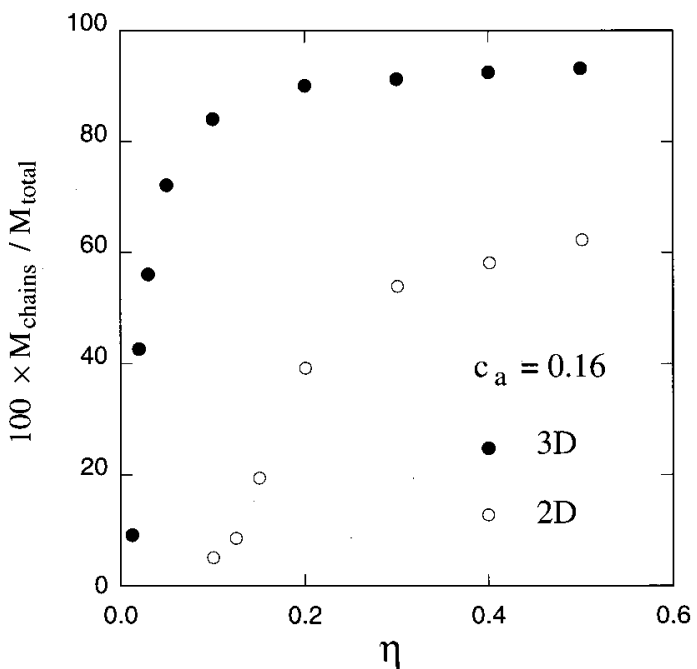

FIG. 12. Fraction of the total system mass in open chains in 2D and 3D as a function of packing fraction $\eta$.

ficient without important assumptions and approximations, but it provides a picture of the mobility of particles in phase space as a function of density and $T .{ }^{20}$

We have compared the mobilities of the three types of particle in the system: regular monomers, and both inert, and active chain terminations. Both types of termination are constrained by one bond rather than two, and they are more mobile than regular particles. However, the most striking difference (at least one order of magnitude, and dependent on $\eta$ and $T$ ) was between active and inert chain terminations. Despite the low frequency of bond interchanges, active particles diffuse much more rapidly than all others and sample a large fraction of the system volume, even at the highest densities and lowest temperatures considered here. This is consistent with the theoretical conjectures that living polymers are far more ergodic than comparable systems at fixed bonding pattern. ${ }^{21}$

\section{THREE-DIMENSIONAL SIMULATIONS}

Simulations for systems in 3D yield analogous results to those for $N_{a}=1,{ }^{12}$ where the tendency to polymerization is much greater in 3D than in 2D. In Fig. 12 we compare the fraction of the total mass represented by open chains in 2D and 3D over a wide density range (for $T=3$ and $c_{a}$ $=0.16 \%$ ). The polymerized fraction is systematically higher in 3D, already reaching $90 \%$ of the total mass at densities of the order of $\eta \sim 0.25$.

The improved sampling provided by several active particles allows us to extend our simulations to lower densities than in Ref. 12 and confirms the existence of a polymerization line in 3D as well. Using the Padé fit described in the previous section, we locate the transition at $\bar{\eta}=0.04 \pm 0.01$, with only slight changes (comparable to the size of the error bar) for $0.1 \%<c_{a}<0.36 \%$. The degree of polymerization depends on $c_{a}$ more than in the 2D case (see Fig. 13), but the average length $\left\langle L_{l}\right\rangle$ still scales approximately as $1 / c_{a}$.

The mass probability distribution can be approximated by the Zimm-Schulz function with an exponent $\gamma$ slightly

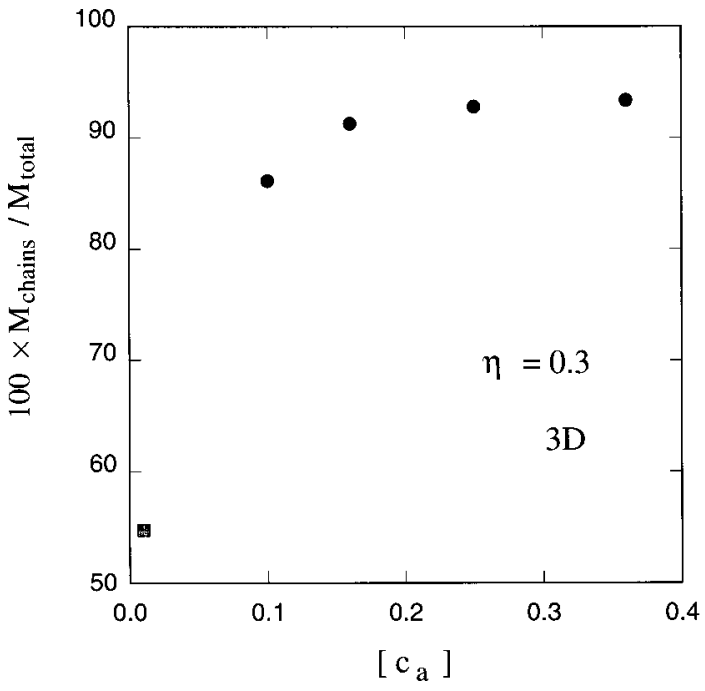

FIG. 13. Solid dots, fraction of the total system mass in open chains in 3D systems as a function of the concentration of active particles $c_{a}$ (in \%). The square shows the result of Ref. 12 for $c_{a}=0.01 \%$. All data refer to $T=3$ and $\eta=0.3$.

lower than in the $2 \mathrm{D}$ case, but a fit to the simpler form $\propto L \exp \left[-L /\left\langle L_{l}\right\rangle\right]$ leads to an exponent that is statistically indistinguishable from the former. These simple properties imply that, within the statistical uncertainties of our results, the distribution functions for the different cases can be folded onto a single curve. The results for 3D systems agree well with those of mean field computations ${ }^{4}$ as well as previous computer simulations for the bond-fluctuation model [see Ref. 8(b)] and the continuum model of Ref. 7. All predict a Zimm-Schulz distribution with an exponent that is close to unity $(\gamma=1.158)$ and lower than in 2D. This agreement does not extend to the case of rings, for which we find $P_{r}(L)$ $\propto L \exp \left[-L /\left\langle L_{r}\right\rangle\right]$, while the studies mentioned above for models in which the number of unsaturated particles is not constrained predict a distribution $P_{r}(L) \propto \exp \left[-L /\left\langle L_{r}\right\rangle\right] /$ $L^{(3 / 2)}$ in $3 \mathrm{D}$, more singular than in $2 \mathrm{D}$.

The degree of polymerization in 3D decreases slightly but monotonically with decreasing $T$, as found in $2 \mathrm{D}$. At $\eta$ $=0.3$ and $c_{a}=0.16 \%$, for example, open chains account for $91.3 \%$ of the total mass for $T=3$, decreasing to $84.8 \%$ at $T$ $=0.6$. We did not observe any clear polymerization transition on changing $T$ alone, so that the polymerization line in the $\eta-T$ plane is a very steep function of $\eta$.

\section{DISCUSSION AND CONCLUDING REMARKS}

Monte Carlo simulations have been performed for systems of LJ particles and harmonic bonds modeling the ring opening polymerization of cyclic polycarbonate oligomers, catalyzed by nucleophilic molecules. Most particles are linked by two harmonic bonds, while $N_{a}$ active particles and an equal number of inert chain terminations (phenol terminations in polycarbonates) form a single bond. Equilibrium polymerization results when at least one of the particles involved is active, so that bonds can be interchanged. Simulations have been carried out in 2D and 3D systems for extended ranges of density and temperature, and we have 
analyzed in detail the effect of different concentrations of active particles in the range $0.10 \% \leqslant c_{a} \leqslant 0.36 \%$. The study extends our previous computations for $c_{a}=0.01 \%$. The necessity of having well converged results has resulted in very long simulations, particularly at low densities, where particle encounters are rare, and at high densities, where all particles are less mobile. A total of approximately 2 years CPU time on modern workstations and servers (Compaq DS20E and XP1000) was needed.

For 2D samples with $0.10 \% \leqslant c_{a} \leqslant 0.36 \%$, the model shows a continuous polymerization transition as the density increases, due to massive increases in the lengths of the open chains. The transition point $\bar{\eta}$ depends only weakly on $c_{a}$, but the steepness of the transition grows rapidly and without apparent upper bound as $c_{a}$ decreases, pointing to a discontinuous transition in the limit of vanishing $c_{a}$. The present results then support our identification of the transition as first order in the limit $c_{a} \rightarrow 0$, studied in our earlier work using $c_{a}=0.01 \%$, and they extend this work to larger concentrations of active particles. At high densities the fraction of the total mass in long chains grows only slightly with increasing $c_{a}$, implying that the average length of chains scales as $1 / c_{a}$. The long open chains coexist with a significant population of rings, whose average length increases slightly upon polymerization and is nearly independent of $c_{a}$. The constant number of chains in our simulations implies that $\left\langle L_{l}\right\rangle$ is proportional to the chain packing fraction $\eta_{l} \cdot{ }^{22}$ In this respect, our model behaves differently from other models of equilibrium polymerization with a variable number of unsaturated particles, for which mean field computations and simulation results ${ }^{23}$ predict $\left\langle L_{l}\right\rangle \propto \eta_{l}^{1 / 2}$.

The signal to noise ratio in the results improves systematically with increasing $c_{a}$, since each open chain provides a (nearly) independent sampling of the polymer properties, and bond interchanges occur more frequently as $N_{a}$ increases. Finite size effects are also reduced, because the competition between different chains reduces their average length, and each chain is progressively less affected by the total system size. These advantages have allowed us to demonstrate the existence of a polymerization transition in 3D, with $\bar{\eta}$ $=0.04 \pm 0.01$. The qualitative features of this transition are very similar to those observed in $2 \mathrm{D}$; it is continuous for every nonvanishing $c_{a}$, becomes sharper with decreasing $c_{a}$, and tends to a discontinuous transition in the $c_{a} \rightarrow 0$ limit.

The improved statistics have allowed us to investigate in detail the probability distribution for the molecular mass rings and open chains in both $2 \mathrm{D}$ and $3 \mathrm{D}$. These distributions are broad, with a standard deviation that is approximately $80 \%$ of the average length for chains, and a monotonically increasing fraction $(\sim 66 \%$ to $200 \%)$ of rings. The large fluctuations in the ring size is due to the presence of a tail in $P_{r}(L)$ that extends to very large rings. A good fit of all probability distributions can be obtained using the ZimmSchulz function with an exponent $\gamma$ in the range 1.2-1.3, and slightly higher in $2 \mathrm{D}$ than in $3 \mathrm{D}$.

The degree of polymerization decreases with decreasing $T$ in all cases, although we did not observe a transition by changing $T$ alone. In agreement with the behavior observed in the simulations for $c_{a}=0.01 \%$, the average potential energy increases as the degree of polymerization increases. The tendency towards the polymeric state is much stronger in $3 \mathrm{D}$ than in $2 \mathrm{D}$.

All these results support our earlier identification ${ }^{12}$ of the entropy of the bond distribution as the driving force for the transition. The bond interchange mechanism allows the system to sample many different bonding configurations, favoring-for sufficiently high density-the long open chains that are much more common than short cyclic bond sequences. ${ }^{24}$ At low density, this entropy contribution is more than compensated by the configurational entropy advantage of small molecules, which may sample independently very large regions of the phase space.

The bond entropy contribution that drives the polymerization of polycarbonates will play a role in several other systems. The polymerization of sulfur, for instance, shares several features with the phase transformation we have observed. A low temperature phase comprising rings is polymerized to long chains by the concentration of radical chain heads that form as $T$ is increased. ${ }^{25}$ However, the dominant role of temperature suggests that potential energy plays a greater role here than in polycarbonates, particularly as it favors the unpolymerized phase that forms again as soon as $T$ is lowered. The role of the entropy associated to the bond distribution in systems other than living polymers is discussed in the Appendix.

\section{ACKNOWLEDGMENTS}

The calculations were performed in the Forschungszentrum Jülich on a Compaq DS20E server and XP1000 workstations provided by the Bundesminister für Bildung und Wissenschaft, Bonn, within the framework of the MaTechKompetenzzentrum "Werkstoffmodellierung" (03N6015). The authors thank A. Milchev and members of the Kompetenzzentrum for helpful discussions.

\section{APPENDIX: RELATED EFFECTS IN OTHER PHYSICAL CONTEXTS}

In the equilibrium polymerization of BPA-PC and other living polymers the entropy of the bonds distribution contributes in an essential way to the total free energy. The achievement of equilibrium implies that bonds can be interchanged fairly easily, and the energy of different bonding configurations cannot differ greatly. Similar effects occur in other systems, even if the energy difference is greater than in BPAPC, and the results of our simulations could have wider validity.

In the $\mathrm{Si}-\mathrm{SiO}_{2}$ binary system ( $\mathrm{Si}$ suboxide) there are widely differing bonding configurations with similar energies, and a recent model ${ }^{26}$ used to describe it has many similarities to ours. The particles have a fixed number of bonds, which can be interchanged during the simulation, and the system eventually reaches a stationary equilibrium distribution. The kinetics of bond interchanges are slow in the $\mathrm{Si}$ suboxide system, which also approaches equilibrium only at high $T$, but there is no obvious analogy of the catalyst in the ring opening of BPA-PC. Annealing of Si suboxide at fairly 
high temperatures results in the segregation of Si nanocrystals, whose high luminescence efficiency makes them interesting for optoelectronic applications. Entropy remains important at the temperatures at which nucleation takes place $(T \geqslant 1200 \mathrm{~K})$, and the bonds contribution may affect the number, size and shape of the microcrystals.

If we focus on the equations governing the evolution of the system, the analogy is even clearer in superfluidity in simple rare gas systems. These are often investigated within the path integral formalism, ${ }^{27}$ in which each atom is represented by a ring of $\mathrm{LJ}$ particles, each corresponding to a different imaginary time slice. ${ }^{28}$ Beads interact with nearest neighbors along the ring via harmonic springs, and the resulting Hamiltonian representing the system in the path integral formulation is very similar to Eq. (1). For indistinguishable particles in the low- $T$ limit, exchange activates bond interchanges and joins "necklaces" into larger rings. Superfluidity, in particular, corresponds to the growth of these units to macroscopic sizes. While the similarities are striking, the superfluid transition shows important differences from the polymerization of BPA-PC. LJ particles interact in the former only at equal imaginary time, the strength of the harmonic bonds is $T$-dependent, and thermodynamic properties are derived from the MC trajectory following quantum mechanical rather than classical rules. The growth of superfluid rings in the path integral simulations is apparently due to the entropy advantage of joining bonds in sequences that do not need to close after a fixed (and short) number of bonds.

${ }^{1}$ For reviews of equilibrium polymerization and related topics, see S. C. Greer, J. Phys. Chem. B 102, 5413 (1998); M. E. Cates and S. J. Candau, J. Phys.: Condens. Matter 2, 6869 (1990).

${ }^{2}$ S. C. Greer, in Adv. Chem. Phys. 94, 261 (1996).

${ }^{3}$ K. M. Zheng and S. C. Greer, Macromolecules 25, 6128 (1992).

${ }^{4}$ L. Schäfer, Phys. Rev. B 46, 6061 (1992); P. D. Gujrati, ibid. 40, 5140 (1989).

${ }^{5}$ See, for instance, Y. Rouault and A. Milchev, Phys. Rev. E 51, 5905 (1995); Y. Rouault and A. Milchev, Europhys. Lett. 33, 341 (1996); Y. Rouault and A. Milchev, Phys. Rev. E 55, 2020 (1997); A. Milchev, Y. Rouault, and D. P. Landau, ibid. 56, 1946 (1997).

${ }^{6}$ I. Carmesin and K. Kremer, Macromolecules 21, 2819 (1988); W. Paul, K. Binder, D. Heermann, and K. Kremer, J. Chem. Phys. 95, 7726 (1991).

${ }^{7}$ A. Milchev, J. P. Wittmer, and D. P. Landau, Phys. Rev. E 61, 2959 (2000).

${ }^{8}$ The following refer to more general models. (a) Semi-rigid polymers have been studied in A. Milchev and D. Landau, Phys. Rev. E 52, 6431 (1995); (b) The role of rings coexisting with open chains has been investigated by J. P. Wittmer, P. van der Schoot, A. Milchev, and J. L. Barrat, J. Chem.
Phys. 113, 6992 (2000); (c) A. Milchev, J. P. Wittmer, and D. P. Landau, Ref. 7.

${ }^{9}$ J. C. Wheeler, S. J. Kennedy, and P. Pfeuty, Phys. Rev. Lett. 45, 1748 (1980); S. J. Kennedy and J. C. Wheeler, J. Chem. Phys. 78, 953 (1983); R. Cordery, Phys. Rev. Lett. 47, 457 (1981); R. G. Petscheck, P. Pfeuty, and J. C. Wheeler, Phys. Rev. A 34, 2391 (1986).

${ }^{10}$ D. J. Brunelle, in Ring-Opening Polymerization: Mechanisms, Catalysis, Structure, Utility, edited by D. J. Brunelle (Hanser, München, Germany, 1993), p. 309

${ }^{11}$ P. Ballone, B. Montanari, and R. O. Jones, J. Phys. Chem. A 104, 2793 (2000); see also P. Ballone and R. O. Jones, ibid. 105, 3008 (2001).

${ }^{12}$ P. Ballone and R. O. Jones, J. Chem. Phys. 115, 3895 (2001).

${ }^{13}$ The LJ potential is set equal to zero for $r \geqslant 3 \sigma$. It is replaced by a third order polynomial for $2.8 \sigma \leqslant r \leqslant 3 \sigma$ such that the potential and its first derivative are continuous throughout.

${ }^{14}$ For simplicity, we exclude isolated particles and rings smaller than trimers.

${ }^{15}$ The restriction to fixed bonding configurations avoids the discontinuous energy jumps that occur on bond interchange following the rules listed in Sec. II. It could be removed by using a more sophisticated model for the bond interchanges or a different MD algorithm.

${ }^{16}$ The choice of equal coefficients for the leading power in the numerator and in the denominator implies that $\left\langle L_{l}(\eta)\right\rangle$ converges to $N_{\text {tot }} / N_{a}=1 / c_{a}$ in the limit of high density.

${ }^{17}$ A comprehensive discussion of size probability distributions for polymers is given in G. C. Berry, Molecular Weight Distribution, in Encyclopedia of Materials Science and Engineering, edited by M. B. Bever (Pergamon, Oxford, 1986), p. 3759. An introduction is given by G. R. Strobl, The Physics of Polymers: Concepts for Understanding their Structures and Behavior (Springer, Berlin, 1996).

${ }^{18} \mathrm{G}$. Porte, in Micelle, Membranes, Microemulsions, and Monolayers, edited by W. M. Gelbart, A. Ben-Shaul, and D. Roux (Springer, Berlin, 1994).

${ }^{19}$ This result is strictly valid only in the case that rings are not allowed; see Ref. 8(b).

${ }^{20}$ Diffusion properties of living polymers have been discussed on the basis of MC mobilities in Y. Rouault and A. Milchev, Europhys. Lett. 33, 341 (1996).

${ }^{21}$ K. Kremer and K. Binder, Comput. Phys. Rep. 7, 259 (1988); N. Madras and A. D. Sokal, J. Stat. Phys. 50, 109 (1988).

${ }^{22}$ Here we divide the total packing fraction $\eta$ into its chain $\left(\eta_{l}\right)$ and ring $\left(\eta_{r}\right.$ ) contributions, $\eta=\eta_{l}+\eta_{r}$

${ }^{23}$ M. E. Cates and S. J. Candau, in Ref. 1.

${ }^{24}$ This argument could provide an explanation of the low probability of large rings observed also in other models; see Ref. 8(b).

${ }^{25}$ M. A. Anisimov, K. I. Kugel, and T. Y. Lisovskaya, High Temp. 25, 165 (1987).

${ }^{26}$ Y. Tu and J. Tersoff, Phys. Rev. Lett. 84, 4393 (2000).

${ }^{27}$ See, for example, D. Chandler, in Theory of Quantum Processes in Liquids, in Les Houches 51, Part 1, Liquids Freezing and Glass Transition, edited by D. Levesque, J.P. Hansen, and J. Zinn-Justin (Elsevier, Amsterdam, 1991), p. 193.

${ }^{28}$ D. M. Ceperley and E. L. Pollock, Phys. Rev. B 39, 2084 (1989). 